\title{
ANÁLISE DA CORRELAÇÃO ENTRE PICO DE TORQUE, DESEMPENHO FUNCIONAL E FROUXIDÃO LIGAMENTAR EM INDIVÍDUOS NORMAIS E COM RECONSTRUÇÃO DO LIGAMENTO CRUZADO ANTERIOR
}

\author{
FUNCTIONAL PERFORMANCE AND KNEE LAXITY IN NORMAL \\ INDIVIDUALS AND IN INDIVIDUALS SUBMITTED TO ANTERIOR \\ CRUCIATE LIGAMENT RECONSTRUCTION
}

Rodrigo Antunes de Vasconcelos ${ }^{1}$, Débora Bevilaqua-Grossi ${ }^{2}$, Antonio Carlos Shimano ${ }^{3}$, Cleber Antonio Jansen Paccola ${ }^{4}$, Tânia Fátima Salvini ${ }^{5}$, Christiane Lanatovits Prado ${ }^{6}$, Wilson A. Mello Junior ${ }^{7}$

\section{RESUMO}

Objetivo: Analisar a correlação entre os déficits musculares dos extensores e flexores do joelho através do pico de torque isocinético com os testes de salto monopodal, frouxidão ligamentar pós-operatória e questionário de função em indivíduos normais e indivíduos submetidos à reconstrução do LCA com enxerto autólogo do tendão patelar e dos tendões flexores. Métodos: Foram recrutados 60 indivíduos, formando três grupos: 20 indivíduos sem lesões no joelho (grupo GC) e dois grupos de 20 indivíduos submetidos à reconstrução do LCA com tendão patelar (grupo GTP) ou tendões flexores (grupo GTF). Resultados: Os resultados demonstraram correlação significativa entre déficits no pico de torque e testes funcionais no torque extensor nos grupos GTF e GC. Não foram observadas correlações significativas entre frouxidão ligamentar pós-operatória e questionário de Lysholm com os testes de salto e déficits no pico de torque. Em relação à diferença entre os grupos, foi observado que o grupo GTP demonstrou maior déficit do torque extensor, menor pontuação no questionário de Lysholm e maior percentagem dos indivíduos com índice de simetria entre membros (ISM) $<90 \%$ em ambos os testes de salto monopodal quando comparado com os demais grupos. Conclusão: Na avaliação funcional de pacientes submetidos à reconstrução do LCA, não é aconselhável a utilização de apenas um instrumento de medida, porque a correlação significativa entre déficit no pico de torque, questionários de função, frouxidão ligamentar e testes de salto não é encontrada em todos os grupos testados.

Descritores - Ligamento cruzado anterior; Joelho; Estudo retrospectivo

\section{ABSTRACT}

Objectives: The aim of this study was to analyze the correlation between deficits in the isokinetic peak torque of the knee extensors and flexors with hop tests, postoperative knee laxity and functional scores in normal and ACL- reconstructed subjects with patellar tendon and hamstring tendon autografts. Methods: Sixty male subjects were enrolled and subdivided into three groups: Twenty subjects without knee injuries (GC group) and two groups of 20 subjects submitted to ACL reconstruction with patellar tendon (GTP group) and hamstrings autograft (GTF group). Results: The results showed significant correlation between knee extensors peak torque and performance in the hop tests for GTF and GC groups. There are no significantly correlations between post op knee laxity and Lysholm score compared with the hop tests and peak torque deficits. Concerning the differences between groups, the GTP group showed greater peak torque deficits in knee extensors, worst Lysholm scores and higher percentage of individuals with lower limb symmetry index (ISM) $<90 \%$ in both hop tests when compared to the other two groups. Conclusion: It is not recommendable to use only one measurement instrument for the functional evaluation of ACL-reconstructed patients, because significant correlation between peak torque, subject's functional score, knee laxity and hop tests were not observed in all groups.

Keywords - Anterior cruciate ligament; Knee; Retrospective study

1 - Doutorando em Ciências Médicas, Área de Concentração Ortopedia, Traumatologia e Reabilitação pela Faculdade de Medicina de Ribeirão Preto FMRP-USP.

2 - Professor Livre-Docente do Departamento de Biomecânica, Medicina e Reabilitação do Aparelho Locomotor da Faculdade de Medicina de Ribeirão Preto FMRP-USP.

3 - Professor Doutor do Departamento de Biomecânica, Medicina e Reabilitação do Aparelho Locomotor da Faculdade de Medicina de Ribeirão Preto FMRP-USP.

4 - Professor Titular do Departamento de Biomecânica, Medicina e Reabilitação do Aparelho Locomotor da Faculdade de Medicina de Ribeirão Preto FMRP-USP.

5 - Professora Titular do Departamento de Fisioterapia da Universidade Federal de São Carlos (UFSCar), São Paulo, Brasil.

6 - Mestranda em Fisioterapia pelo Departamento de Fisioterapia da Universidade Federal de São Carlos (UFSCar), São Paulo, Brasil.

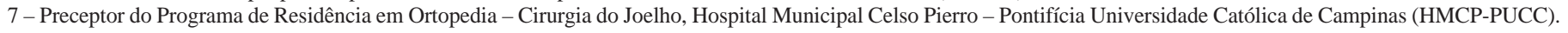

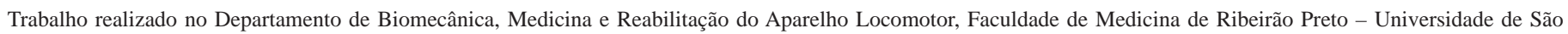
Paulo (FMRP-USP). Estudo realizado com apoio financeiro proporcionado pela Capes.

Correspondência: Rua Dom Bosco, 187, Bairro Taquaral - Campinas, SP - CEP: 13076-060 - E-mail: rodvasconcelos@hotmail.com 


\section{INTRODUÇÃO}

A lesão ou ruptura do ligamento cruzado anterior (LCA) em atletas ou indivíduos fisicamente ativos é muito comum na prática clinica. Estudos epidemiológicos demonstram incidência aproximada de 80.000 lesões por ano ${ }^{(1)}$. Influenciado por estes fatores, houve enorme crescimento no número de trabalhos publicados nos últimos 15 anos, com pesquisas enfocando a anatomia, biomecânica, cirurgia de reconstrução e técnicas de reabilitação do LCA. O avanço das pesquisas nesse campo da ortopedia diminuiu consideravelmente o tempo de retorno ao esporte de atletas submetidos à reconstrução do $\mathrm{LCA}^{(2)}$. Essa redução no tempo de retorno ao esporte se deve principalmente ao avanço das técnicas cirúrgicas, fundamentalmente com a utilização da artroscopia, propiciando técnicas menos invasivas e diminuindo complicações no pós-operatório, como artrofibrose, melhora das técnicas de fixação do enxerto, possibilitando a mobilização precoce da articulação do joelho seguida da utilização de reabilitação acelerada ${ }^{(3)}$ Como a lesão do LCA ocorre em sua maioria em indivíduos fisicamente ativos, uma pergunta frequente é direcionada a ortopedistas e fisioterapeutas envolvidos nessa especialidade: “Quando retornarei ao esporte após a cirurgia?”.

A literatura atual não demonstra consenso a respeito do tempo ideal de retorno ao esporte, uma vez que há grande variedade de trabalhos publicados demonstrando retorno ao esporte em fases extremamente precoces, seis semanas $^{(4)}$, ou em fases tardias, a partir de nove meses ${ }^{(5)}$. Mesmo com essa discrepância encontrada na literatura, todos os autores que publicaram a respeito de tempo de retorno ao esporte usam protocolos específicos de avaliação funcional para tomada de decisão.

Para retorno seguro ao esporte competitivo ou atividade física sem restrições após reconstrução do LCA, são necessários critérios objetivos e subjetivos relacionados com a estabilidade clinica do joelho ${ }^{(6)}$, amplitude de movimento $^{(7)}$, hipotrofia dos músculos da coxa ${ }^{(8)}$, questionários numéricos de função ${ }^{(9)}$, testes funcionais que envolvam desde saltos monopodais a testes de agilidade ${ }^{(10)}$ e avaliações do desempenho muscular ${ }^{(11)}$. A avaliação do desempenho muscular, especificamente dos extensores e flexores do joelho, pode ser realizada por meio de testes isotônicos, isocinéticos ou isométricos, através da execução de contrações concêntricas, excêntricas ou isométricas $^{(12)}$. Entre todas as alternativas previamente citadas, a avaliação dos déficits musculares, que se caracteriza pela diferença significativa de desempenho muscular entre membro lesado ou dominante comparado com o lado saudável ou contralateral, é realizada através dinamôme- tros isocinéticos computadorizados, sendo considerados os mais apropriados para coleta de dados devido a sua objetividade, segurança e reprodutibilidade em avaliações ${ }^{(13)}$. Além disso, estudos que utilizam dinamômetros isocinéticos fornecem referências para progressão das fases da reabilitação no pós-operatório ${ }^{(14)}$ e para critério de retorno ao esporte sem restrições ${ }^{(15)}$. Porém, pesquisas a respeito de critério de retorno ao esporte têm gerado grande controvérsia na literatura a respeito da validade dos dinamômetros isocinéticos em se correlacionarem com diferentes testes funcionais que simulam o gesto esportivo $^{(16,17)}$. Vários trabalhos observaram correlação significativa entre pico de torque e desempenho em testes de salto monopodal e questionário de função ${ }^{(16,18)}$. No entanto, outros pesquisadores argumentam que os dinamômetros isocinéticos são exames físicos realizados em cadeia cinética aberta, portanto, com diferenças importantes em relação ao movimento do gesto esportivo, e que testes de salto monopodal reproduziriam melhor os déficits funcionais encontrados ${ }^{(19)}$.

Portanto, este trabalho tem os seguintes objetivos:

1) Investigar a correlação entre os déficits encontrados no pico de torque isocinético com déficits encontrados nos testes de salto monopodal, questionário de Lysholm e com a frouxidão ligamentar pós-operatória nos indivíduos submetidos à reconstrução do LCA.

2) Analisar os déficits musculares dos extensores e flexores do joelho através do pico de torque isocinético em indivíduos normais e indivíduos submetidos à reconstrução do LCA com enxerto autólogo do tendão patelar e dos tendões flexores (semitendinoso e grácil).

3) Avaliar os déficits encontrados em duas modalidades de salto monopodal em indivíduos normais e indivíduos submetidos à reconstrução do LCA com enxerto autólogo do tendão patelar e dos tendões flexores (semitendinoso e grácil).

\section{MÉTODOS}

Foram convidados através de comando verbal 60 indivíduos com idade entre 18 e 45 anos. Os voluntários foram divididos em três grupos. $\mathrm{O}$ primeiro foi denominado de grupo controle (GC), consistindo de 20 voluntários sem história prévia de lesões no joelho. O segundo grupo, denominado grupo tendão patelar (GTP), foi formado por 20 voluntários submetidos à reconstrução do ligamento cruzado anterior com enxerto autólogo do tendão patelar. O terceiro grupo foi constituído por 20 voluntários submetidos à reconstrução do ligamento cruzado anterior com enxerto autólogo dos tendões flexores (semitendinoso e grácil); esse grupo foi denominado 
grupo tendões flexores (GTF). Todos os voluntários dos grupos GTP e GTF foram recrutados após ter completado o mínimo de seis meses de pós-operatório, sendo este estudo retrospectivo. Todos os integrantes dos três grupos participavam de atividades esportivas recreativas sem fim competitivo. Os procedimentos cirúrgicos foram realizados pelos cirurgiões ortopedistas do grupo de cirurgia do joelho do Hospital Municipal Celso Pierro - Pontifícia Universidade Católica de Campinas. As características gerais dos participantes dos três grupos, detalhes do status pós-operatório dos grupos GTP e GTF estão descritos na tabela 1 .

Tabela 1 - Características gerais e do status pós-operatório dos voluntários distribuídos nos três grupos analisados GC, GTP e GTF

\begin{tabular}{|c|c|c|c|}
\hline Variáveis & Grupo GC & Grupo GTP & Grupo GTF \\
\hline Amostra (N) & 20 & 20 & 20 \\
\hline Idade (anos) & $24,95( \pm 5,18)$ & $32,6( \pm 7,76)$ & $27,55( \pm 6,88)$ \\
\hline Altura (cm) & $175,1( \pm 7,35)$ & $176,2( \pm 8,48)$ & $179,5( \pm 8,99)$ \\
\hline Peso (kg) & $75,8( \pm 8,7)$ & $85,5( \pm 14,3)$ & $79,9( \pm 8,7)$ \\
\hline ADM flexão ND e L & $143,5( \pm 3,5)$ & $137,6( \pm 7,46)$ & $136,9( \pm 3,9)$ \\
\hline ADM flexão D e L & $143,5( \pm 3,5)$ & $137,5( \pm 6,6)$ & $137,8( \pm 3,9)$ \\
\hline $\begin{array}{l}\text { ADM extensão } \\
\text { ND e L }\end{array}$ & $-0,2( \pm 3,49)$ & $-1,2( \pm 4,6)$ & $-0,15( \pm 2,15)$ \\
\hline $\begin{array}{l}\text { ADM extensão } \\
\text { D e NL }\end{array}$ & $-0,1( \pm 3,17)$ & $1,2( \pm 4,07)$ & $1,25( \pm 4,7)$ \\
\hline Perimetria $(\mathrm{a} 15 \mathrm{~cm})$ & $0,8( \pm 0,6)$ & $1,42( \pm 1,1)$ & $1,35( \pm 1,3)$ \\
\hline Escala Tegner & 5,5 & 6,2 & 6,7 \\
\hline Fixação femoral & 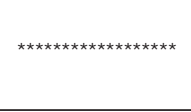 & Interferência 20120 & $\begin{array}{c}\text { Endobutton } 5120 \\
\text { Transverso } 8120 \\
\text { Interferência } 7120 \\
\end{array}$ \\
\hline Fixação tibial & 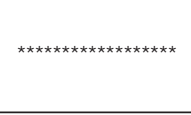 & Interferência 20120 & $\begin{array}{l}\mathrm{PC}+\mathrm{AM} 10120 \\
\text { Int + Blount } 2120 \\
\text { Interferência } 8120\end{array}$ \\
\hline Tempo P.O (meses) & 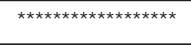 & $8,5( \pm 5,45)$ & $8,5( \pm 4,00)$ \\
\hline Reabilitação Superv & 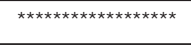 & 10120 & $15 \backslash 20$ \\
\hline Reabilitação Orient. & 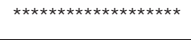 & 10120 & 5120 \\
\hline \multicolumn{4}{|c|}{$\begin{array}{l}\text { Legenda: ND e } \mathrm{L}=\text { não dominante e lado lesado; } \mathrm{D} \text { e } \mathrm{L}=\text { dominante e lesado; superv = su } \\
\text { pervisionada; orient = orientada; } \mathrm{PC}+\mathrm{AM}=\text { parafuso cortical + arruela metálica; int + blount } \\
\text { parafuso de interferencia + blount ; GC = grupo controle ; GTP = grupo tendão patelar; GTF } \\
\text { grupo tendões flexores. }\end{array}$} \\
\hline Fonte : FMRP-USP & & & \\
\hline
\end{tabular}

Os critérios de inclusão do grupo (GC) foram: ausência de histórias prévias de lesões no joelho de qualquer natureza, ausência de desordens neuromusculares, diferenças de frouxidão ligamentar entre joelhos menor do que 3mm avaliados pelo KT 1000. Os critérios de inclusão do grupo GTP e GTF foram: seis meses de pós- operatório completos, ausência de sinais inflamatórios, marcha normal, ausência de desordens neuromusculares e sem queixas de instabilidade em Avds. Os critérios de exclusão para todos os grupos foram: histórico de lesões ligamentares bilaterais, cirurgias de reconstrução ligamentar de qualquer na- tureza prévias no joelho, fraturas de qualquer natureza nos membros inferiores, rupturas ligamentares combinadas, osteoartrose avançada nas articulações femoropatelar ou tibiofemoral com evidente desvio de eixo articular

Todos os voluntários receberam instruções detalhadas por escrito sobre como seria o teste e assinaram termo de consentimento livre e esclarecido, concordando em participar do estudo, sendo o projeto aprovado pelo Comitê de Ética do Hospital das Clínicas da Faculdade de Medicina de Ribeirão Preto-Universidade de São Paulo, oficio no 7375/2007

A versão validada para a língua portuguesa da avaliação subjetiva de Lysholm $^{(20)}$ foi utilizada para caracterizarmos funcionalmente a amostra. Para avaliação do nível esportivo dos voluntários nos três grupos foi utilizada a Escala de Tegner e Lysholm ${ }^{(21)}$.

O artrômetro KT 1000 (MEDmetric, San Diego, CA) foi utilizado para avaliar todos os grupos. No grupo GC, o equipamento determinou o critério de inclusão e exclusão dos participantes no estudo. Nos grupos GTP e GTF, o KT 1000 foi utilizado para determinar a estabilidade clínica do joelho operado.

Para a coleta do pico de torque isocinético foi utilizado o dinamômetro isocinético computadorizado (Biodex Multi-joint System 3 Pro), pertencente ao Laboratório de Avaliação e Intervenção em Ortopedia e Traumatologia (LAIOT), do curso de pós-graduação em Fisioterapia da Universidade Federal de São Carlos (UFSCar).

\section{Procedimentos}

Inicialmente, todos os participantes foram submetidos a uma avaliação clinica. Nesta, além da coleta dos dados pessoais, os voluntários foram submetidos ao exame físico, que compreende verificação da amplitude do movimento passiva (ADM), perimetria de coxas e preenchimento do questionário de Lysholm e Tegner. Após a avaliação clínica, os voluntários foram submetidos ao exame de artrometria (KT 1000), através do teste máxima manual (TMM). Foram realizados três TMM e o maior valor foi anotado na ficha de avaliação clinica (Figura 1A). Nos grupos GTP e GTF, o joelho não lesado sempre foi testado primeiro e, no grupo controle, o joelho não dominante. Todos os exames de artrometria foram realizados pelo primeiro autor deste trabalho, sendo a confiabilidade teste-reteste do examinador publicada anteriormente ${ }^{(22)}$. Após o teste máxima manual, todos os voluntários realizaram um aquecimento de cinco minutos em uma bicicleta ergométrica, seguido de três séries de 30 segundos de alongamentos tradicionais para quadríceps e isquiotibiais. 


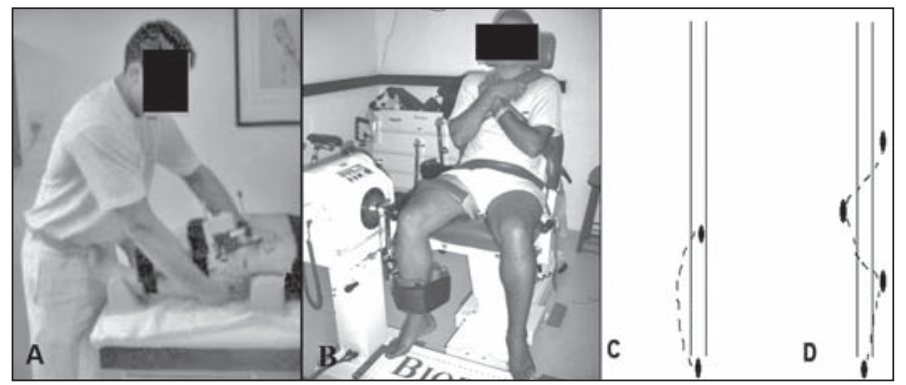

Figura 1 - A) Teste máxima manual utilizando o KT 1000; B) posicionamento dos voluntários* para coleta do pico de torque isocinético dos extensores e flexores do joelho; $\mathrm{C}$ ) teste de salto monopodal a distância; e D) teste de salto triplo monopodal a distância (adaptado do original Noyes et al ).

Após o aquecimento, os pacientes foram posicionados na cadeira do dinamômetro isocinético e estabilizados no tronco, pelve e quadril por cintas de contenção para evitar compensações durante o exame (Figura 1B). Os voluntários foram instruídos a segurar seus braços cruzados sobre o tórax para isolar o momento extensor e flexor do joelho.

Para a avaliação do torque isocinético, foi elaborado protocolo com duas velocidades, $60 \%$ s e $180 \%$ s. Na velocidade de $60 \%$, os voluntários realizaram duas séries de cinco movimentos de extensão e flexão do joelho, e a $180 \%$ s, duas séries de 15 repetições; ambas as velocidades foram realizadas com os joelhos percorrendo entre os ângulos de $0^{\circ}$ e $90^{\circ}$ o mais rápido e forte possível, com intervalos entre séries de 90 segundos. A primeira série foi utilizada para familiarização do paciente com o teste isocinético. Durante o teste, o paciente recebeu comando verbal constante para realizar o exame. $\mathrm{O}$ exame para todos os testes foi iniciado com a perna não dominante no grupo GC e na perna contralateral nos grupos GTP e GTF. Foram considerados déficits significativos diferenças do lado lesado acima de $15 \%$ em relação ao lado contralateral nos grupos GTP e GTF e lado não dominante comparado com o lado dominante no grupo GC. O valor de referencia de $15 \%$ foi baseado em trabalho de Kvist et $\mathrm{al}^{(23)}$, que fornece recomendações relacionadas ao déficit máximo aceito para retorno à atividade esportiva sem restrições após reconstrução do LCA.

\section{Testes de salto monopodal (hop tests)}

Para avaliação dos testes de salto monopodal (hop tests), os pacientes foram instados a escolher entre dois envelopes pequenos que continham ou o teste monopodal a distância ou o teste triplo unipodal a distância, sendo este o método de randomização da ordem de execução dos dois testes de salto monopodal. Para a realização dos saltos, são colocadas no solo duas fitas adesivas com $6 \mathrm{~m}$ de comprimento e separadas entre si há a distância de $15 \mathrm{~cm}$.
Após a escolha da ordem dos saltos, foram dadas instruções verbais a respeito da forma correta de execução do teste. O objetivo do teste de salto monopodal a distância é saltar o mais longe possível com apenas um membro e aterrissar com o mesmo (Figura 1C). O objetivo do teste de salto monopodal triplo cruzado a distância é realizar três saltos em ziguezague o mais longe possível, cruzando as duas linhas paralelas, apenas com um membro inferior (Figura 1D). Após as instruções, os voluntários foram posicionados no início da fita adesiva colocada no solo para referência e orientação. Foi permitido apenas o apoio de um membro inferior para o início do teste. Os indivíduos foram orientados a deixar os membros superiores posicionados à livre escolha durante a execução do teste.

Não foi realizada familiarização do teste com o objetivo de observar melhor as diferenças entre os grupos.

Para obtenção dos déficits apresentados pelos voluntários ao executarem as duas modalidades de salto, foi utilizado como referência o trabalho de Noyes et $\mathrm{al}^{(24)}$, que utilizam a seguinte equação:

$$
\begin{gathered}
\text { ISM }=\frac{\text { distância lado não dominante }}{\text { distância lado dominante }} \times 100 \\
\text { ISM }=\frac{\text { distância membro lesado }}{\text { distância membro contralateral }} \times 100 \\
\text { GTP e GTF }
\end{gathered}
$$

ISM - índice de simetria do membro inferior , GC - grupo controle, GTP - grupo tendão patelar, GTF- grupo tendões flexores

Onde ISM significa índice de simetria do membro inferior, para demonstrar déficit significativo entre membros; o lado lesado ou o joelho com menor pontuação no grupo controle deverá demonstrar distância menor do que $90 \%$ do valor do lado contralateral ${ }^{(25)}$.

\section{ANÁLISE ESTATÍSTICA}

Para realizar a comparação intergrupos: GC, GTP e GTF nos testes isométricos, isocinéticos, questionário de Lysholm e artrometria, foi utilizada análise de variância (ANOVA) com teste de post hoc de Bonferroni tendo como valor de $\mathrm{p}<0,05$. Para efetuar a análise da correlação entre déficits no pico de torque isométrico e isocinético e desempenho nos testes de salto, frouxidão ligamentar e questionário de Lysholm intragrupos, foi utilizado o cálculo do coeficiente de correlação de Pear- 
son. Para realizar a comparação entre os indivíduos dos grupos GTP e GTF que tiveram translação anterior da tíbia maior que 3mm entre joelhos, bem como a comparação desses indivíduos realizando testes de salto, foi utilizado o teste $t$ de Student para amostras independentes, considerando valor de $\mathrm{p}<0,05$.

Foi realizada, adicionalmente, comparação quantitativa em relação à percentagem de indivíduos nos três grupos que apresentaram déficit no torque extensor maior que $15 \%$ entre joelhos nas duas velocidades angulares; do ponto de vista de critério de retorno ao esporte, não estariam com simetria entre membros adequada; e comparação em relação à percentagem de indivíduos nos três grupos que apresentaram ISM $<90 \%$ nas duas modalidades de testes de salto monopodal; do ponto de vista de critério de retorno ao esporte, não estariam com simetria entre membros adequada. Foi utilizado cálculo de regressão logística, cuja variável independente sempre foi grupo (três grupos) e a variável resposta, cada um dos testes (torque extensor, flexor e testes de salto monopodal), considerando valor de $\mathrm{p}<0,05$.

\section{RESULTADOS}

Na tabela 1 podem-se observar os dados descritivos das características dos participantes do estudo, referentes a idade, peso e altura. Podem-se observar também valores referentes ao status pós-operatório entre os grupos GTP e GTF demonstrando valores similares de ADM de flexão e extensão, perimetria, tempo médio de pós-operatório (8,5 meses), principalmente devido ao fato de que os participantes do grupo GTP e GTF foram recrutados em ordem cronológica através da consulta do banco de dados de cirurgias realizadas no Hospital e Maternidade Celso Pierro - Pontifícia Universidade Católica de Campinas (HMCP-PUCC), uma vez sendo este estudo no formato retrospectivo. Um aspecto importante neste estudo foi a intenção de incluir os pacientes o mais próximo possível do tempo de alta mínimo comumente utilizado na literatura, sendo entre seis e nove meses. No grupo GTP, 80\% (16/20) dos indivíduos estavam nessa fase e $85 \%$ (17/20) no grupo GTF. Em relação às lesões associadas no grupo GTP, foram observadas lesões condrais tibiofemoral + menisco medial $(1 / 20)$, lesão menisco medial (4/20), lesão menisco medial e lateral (1/20). No grupo GTF lesão condral + menisco medial $(2 / 20)$, lesão condral tibiofemoral $(1 / 20)$, lesão menisco medial (4/20). O nível de participação esportiva nos indivíduos nos três grupos permaneceu entre 5,5 a 6,7 na escala de Tegner.

\section{Artrometria e questionário de Lysholm}

Na avaliação da frouxidão ligamentar intergrupos, o grupo GC demonstrou valores de translação anterior da tíbia (TAT) entre joelhos significativamente menores ( $\mathrm{p}<$ 0,001 ) que os grupos GTP e GTF (Tabela 2). Não houve diferenças estatisticamente significativas entre os grupos GTP e GTF (Tabela 2). Na avaliação funcional utilizando-se do questionário de Lysholm, houve diferenças estatisticamente significativas entre os grupos; o grupo GC apresentou pontuação significativamente maior comparada com a dos grupos GTP e GTF (p < 0,001) (Tabela 2). Na comparação entre os grupos GTP e GTF, este demonstrou pontuação significativamente maior que aquele $(p<0,001)$.

Tabela 2 - Comparação intergrupos referentes à média $(\mathrm{dp})$ do índice de simetria (ISM) dos valores dos testes de salto monopodal, frouxidão ligamentar e questionário de Lysholm utilizando-se de análise de variância ANOVA com teste post hoc de Bonferroni

\begin{tabular}{l|c|c|c|c|c|c}
\hline Teste de salto & $\begin{array}{c}\text { GC } \\
\mathbf{N}=\mathbf{2 0}\end{array}$ & $(\mathbf{d p})$ & $\begin{array}{c}\text { GTP } \\
\mathbf{N = 2 0}\end{array}$ & (dp) & $\begin{array}{c}\text { GTF } \\
\mathbf{N}=\mathbf{2 0}\end{array}$ & (dp) \\
\hline Salto monopodal & $94,62^{*}$ & $(3,63)$ & 78,35 & $(12,55)$ & 86,15 & $(18,51)$ \\
\hline Triplo monopodal & $97,11^{*}$ & $(2,33)$ & 80,46 & $(10,28)$ & 86,93 & $(16,20)$ \\
\hline Kt $1000(\mathrm{~mm})$ & $0,7^{\star *}$ & $(0,49)$ & 2.94 & $(2,26)$ & 2,89 & $(1,98)$ \\
\hline Lysholm & $100^{*}$ & $(.0)$ & $87.4 \dagger$ & $(12,9)$ & 93,8 & $(6,14)$ \\
\hline
\end{tabular}

Legenda: GC - grupo controle; GTP - grupo tendão patelar; GTF - grupo tendões flexores;

* Grupo controle com valores significativamente maiores que os do grupo GTP e GTF $(p<0,001)$

** Grupo controle com valores significativamente menores que os do grupo GTP e GTF $(p<0,001)$ † Grupo GTP com valores significativamente menores que os do grupo GC e GTF $(p<0,001)$ Fonte : FMRP-USP

\section{Pico de torque isocinético}

Na análise intergrupos, os déficits encontrados no torque extensor no grupo GC foram significativamente menores $(\mathrm{p}<0,001)$ no pico de torque isocinético a $60^{\circ} / \mathrm{s}$ $(7,71 \pm 4,21)$ e $180 \%$ s $(5,85 \pm 3,89)$ quando comparados com os do grupo GTP $(32,30 \pm 14,30),(22,63 \pm 10,61)$ e com os do GTF $(18,70 \pm 17,79)$ e $(15,83 \pm 15,26)$, respectivamente. Na comparação entre os grupos GTP e GTF, o primeiro demonstrou déficits maiores nos dois testes de força, significativos ( $\mathrm{p}<0,001$ ), no teste isocinético a $60 \%$ s $(32,30 \pm 14,30)$ contra $(18,70 \pm 17,79)$ no grupo GTF. Pode ser observado no gráfico 1 uma comparação quantitativa em relação à percentagem de indivíduos nos três grupos que apresentaram déficit no torque extensor maior que $15 \%$ entre joelhos nas duas velocidades angulares; do ponto de vista de critério de retorno ao esporte, não estariam com simetria adequada entre membros.

O grupo GTP apresentou significativamente maior número de indivíduos dentro desse parâmetro quando comparado com o grupo GC nas velocidade de teste de $60 \%$ s e $180 \%$ s $(p=0,02)$ e quando comparado com o grupo GTF nas velocidades de $60^{\circ} / \mathrm{s}(\mathrm{p}=0,01)$ e $180 \% \mathrm{~s}(\mathrm{p}=0,05)$. Na 


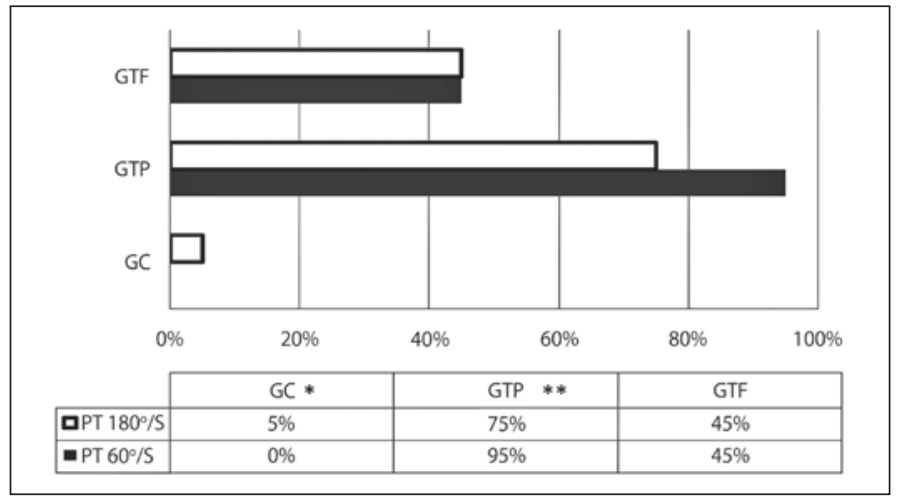

Gráfico 1 - Comparação intergrupos referente à percentagem de pacientes que demonstraram déficits do torque extensor maiores que $15 \%$ entre joelhos no pico de torque isocinético a $60 \% / \mathrm{s}$ (PT 60\%/s) e 180\%/s (PT 180\%/s)

Legenda: * GC x GTP PT 180\%/s ( $p=0,04)$ e GC x GTF PT 180\% ( $p=0,01)$; GC x GTP PT 60\%/s e GC $\times$ GTF PT $60 \%(p=0,02){ }^{* *}$ GTP $\times$ GTF PT $180 \% / s(p=0,05)$ e GTP $\times$ GTF PT $60 \% / s(p=0,01)$ Fonte: FMRP-USP

análise dos déficits no pico de torque flexor, o grupo GC apresentou déficits de $(5,05 \pm 5,13)$ no isocinético a $60 \%$ e $(6,09 \pm 3,85)$ a $180 \%$ s. No grupo GTP foram observados valores de déficits de $(4,0 \pm 12,22)$ no teste isocinético a $60 \%$ e $(2,51 \pm 13,49)$ a $180 \%$ s. O grupo GTF apresentou déficits de $(8,99 \pm 10,01)$ no isocinético a $60 \%$ e $(7,20$ $\pm 9,85)$ a $180^{\circ}</$ s. Não houve diferença estatisticamente significativa entre os três grupos avaliados ( $\mathrm{p}>0,05)$. $\mathrm{Na}$ análise quantitativa entre os grupos considerando o torque flexor, não houve diferença estatisticamente significativa entre a percentagem de indivíduos que apresentaram déficit > 15\% entre joelhos (Gráfico 2).

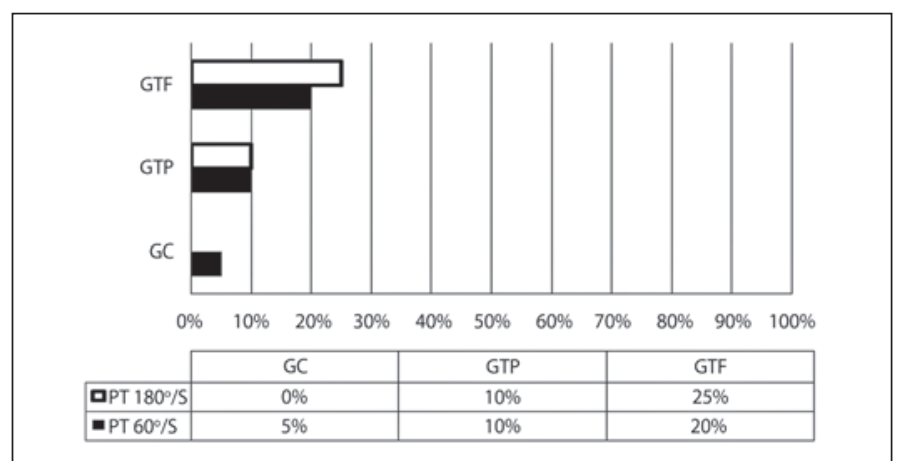

Gráfico 2 - Comparação intergrupos referente à percentagem de pacientes que demonstraram déficits do torque flexor maiores que $15 \%$ entre joelhos no pico de torque isocinético a $60 \%$ s (PT $60 \%$ s) e $180 \%$ s (PT $180 \%$ s)

\section{Testes de salto monopodal (hop tests)}

Na avaliação intergrupos utilizando-se do método ANOVA, houve diferenças estatisticamente significativas no desempenho realizando os dois saltos monopodais (salto monopodal a distância e salto triplo mo- nopodal a distância) com valores médios de índice de simetria de membros inferiores (ISM) significativamente maiores no grupo GC quando comparados com os dos grupos GTP e GTF ( $<<0,001$ ) (Tabela 2). O grupo GTP demonstrou valores médios de ISM menores que os dos grupos GC e GTF, porém, não houve diferenças significativas entre os grupos GTP e GTF em ambos os testes de salto (Tabela 2). No gráfico 3 realizamos a comparação intergrupos referentes ao número de indivíduos dentro de cada grupo que apresentou valor de ISM menor que 90\%. Um número significativamente maior de indivíduos do grupo GTP não atingiu valores ideais de ISM ( $\geq 90 \%$ ) para retorno ao esporte quando comparados com os dos grupos GC e GTF nos testes de salto monopodal e salto triplo monopodal (Gráfico 3).

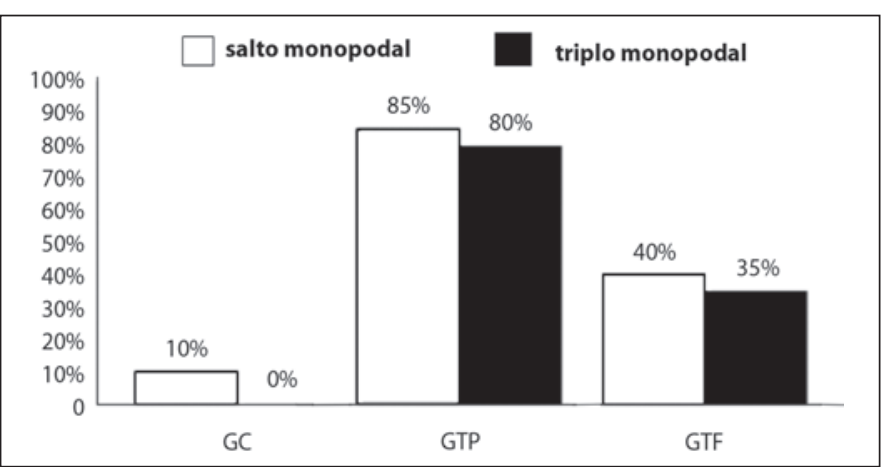

Gráfico 3 - Comparação intergrupos referente à percentagem de indivíduos que demonstraram índice de simetria entre membros inferiores (ISM) menor que $90 \%$ nos testes de salto monopodal . GC $(N=20)$, GTP $(N=20)$ e GTF $(N=20)$

Fonte : FMRP-USP

Legenda: * GC x GTP salto monopodal $(p<0,001)$ e GC $\times$ GTF salto monopodal $(p<0,04)$; GC $\times$ GTP salto triplo monopodal e salto triplo monopodal $(p<0,02)$

** GTP x GTF salto monopodal $p=0,06$ e triplo monopodal $(p=0,06)$

Fonte: FMRP-USP

\section{Correlação entre pico de torque, testes de salto, frouxidão ligamentar e questionário de Lysholm}

$\mathrm{Na}$ análise intragrupos da correlação entre pico de torque flexor e frouxidão ligamentar mensurada através do KT 1000, não houve correlação estatisticamente significativa na grande maioria dos testes de força utilizados (Tabela 3). Foi observada apenas no grupo GTP correlação negativa significativa, porém moderada, entre déficit no pico de torque flexor no teste isocinético a $60^{\circ} / \mathrm{s} \mathrm{e}$ frouxidão ligamentar $(r=-0,49 ; p=0.03)$. Nos demais grupos e testes não houve correlação significativa entre pico de torque flexor $\mathrm{x}$ testes de salto e com a pontuação obtida no questionário de Lysholm (Tabela 3).

$\mathrm{Na}$ análise intragrupos a respeito da correlação entre déficits no pico de torque extensor e frouxidão ligamentar, não houve correlação significativa em nenhum dos grupos (Tabela 4). A mesma situação foi observada 
Tabela 3 - Coeficiente de correlação de Pearson entre déficits de pico de torque flexor isocinético a 60\%/s (PT 60\%) e 180\%/s (PT $180 \%$ s) e salto monopodal, frouxidão ligamentar e questionário de Lysholm nos grupos GC ( $n=20)$, GTP $(n=20)$ e GTF $(n=20)$

\begin{tabular}{|c|c|c|c|c|c|c|}
\hline Teste & $\mathrm{GC}(r)$ & $\mathrm{p}$ & GTP (r) & $\mathrm{p}$ & GTF (r) & $\mathrm{p}$ \\
\hline PT $60 \%$ s salto monopodal & $-0,31$ & 0,19 & 0,06 & 0,81 & $-0,28$ & 0,23 \\
\hline PT $180^{\circ}$ s $x$ salto monopodal & 0,08 & 0,75 & 0,06 & 0,82 & $-0,07$ & 0,77 \\
\hline PT $60 \%$ s $x$ salto triplo monopodal & 0,12 & 0,61 & $-0,04$ & 0,86 & $-0,31$ & 0,18 \\
\hline PT $180^{\circ}$ /s $\times$ salto triplo monopodal & 0,10 & 0,66 & $-0,06$ & 0,82 & $-0,22$ & 0,35 \\
\hline PT $60 \%$ s x kt 1000 max man & 0,32 & 0,17 & $-0,49$ & 0,03 & $-0,14$ & 0,57 \\
\hline PT $180^{\circ} / \mathrm{s}$ x kt 1000 max man & 0,19 & 0,41 & 0,02 & 0,94 & $-0,24$ & 0,31 \\
\hline PT $60 \%$ s x Lysholm & Var const & & 0,28 & 0,26 & 0,07 & 0,78 \\
\hline PT 180 \% x x Lysholm & Var const & & $-0,07$ & 0,79 & $-0,10$ & 0,68 \\
\hline
\end{tabular}

Legenda: GC - grupo controle; GTP - grupo tendão patelar; GTF - grupo tendões flexores ; Var const - variável constante * correlação estatisticamente significativa $(p<0,05)$

Fonte: FMRP-USP

quando comparados pico de torque extensor e pontuação obtida no questionário de Lysholm, não sendo observadas correlações significativas entre os grupos (Tabela 4). Na avaliação dos déficits no pico de torque extensor com o desempenho nos testes de salto, houve correlações negativas estatisticamente significativas entre pico de torque extensor no teste isocinético a $60 \%$ s comparado com o teste de salto monopodal no grupo GC $(r=-0,53$; $p<$ 0,02). Não houve correlação significativa entre déficits no pico de torque extensor com o desempenho nos testes de salto no grupo GTP. No grupo GTF houve correlações negativas estatisticamente significativas entre déficit do pico de torque extensor a $60 \%$ e salto monopodal a distância ( $\mathrm{r}=-0,90 ; \mathrm{p}<0,001)$ e salto triplo cruzado a distancia $(r=-0,78 ; \mathrm{p}<0,001)$ e entre pico de torque extensor a $180 \%$ s e salto monopodal a distância $(\mathrm{r}=-0,87$; $\mathrm{p}<$ $0,001)$ e salto triplo a distância $(r=-0,78 ; \mathrm{p}<0,001)$.

Tabela 4 - Coeficiente de correlação de Pearson entre déficits de pico de torque extensor, isocinético a $60 \%$ s (PT 60\%/s) e 180\%/s (PT 180\%/s) e hop tests, frouxidão ligamentar e questionário de Lysholm nos grupos GC $(n=20)$, GTP $(n=20)$ e GTF $(n=20)$

\begin{tabular}{|c|c|c|c|c|c|c|}
\hline Teste & $\mathrm{GC}(\mathrm{r})$ & $\mathrm{p}$ & GTP $(r)$ & $\mathrm{p}$ & GTF (r) & $\mathrm{p}$ \\
\hline PT $60^{\circ} / \mathrm{s} \times$ salto monopodal & $-0,53$ & $0,02^{*}$ & $-0,43$ & 0,06 & -0.90 & $<0,001^{*}$ \\
\hline PT $180^{\circ} / \mathrm{s} \times$ salto monopodal & 0,03 & 0,90 & $-0,34$ & 0,14 & $-0,87$ & $<0,001^{*}$ \\
\hline $\begin{array}{l}\mathrm{PT} 60^{\circ} / \mathrm{s} \times \text { salto triplo } \\
\text { monopodal }\end{array}$ & $-0,18$ & 0,46 & $-0,21$ & 0,38 & $-0,78$ & $<0,001^{*}$ \\
\hline $\begin{array}{l}\text { PT } 180 \% / s \times \text { salto triplo } \\
\text { monopodal }\end{array}$ & $-0,04$ & 0,86 & $-0,32$ & 0,17 & $-0,78$ & $<0,001^{\prime}$ \\
\hline PT $60^{\circ} / \mathrm{s} \times \mathrm{KT} 1000$ max man & 0,33 & 0,16 & $-0,06$ & 0,82 & 0,12 & 0,62 \\
\hline PT 180\% x K KT 1000 max man & -.18 & 0,44 & 0,05 & 0,85 & 0,25 & 0,29 \\
\hline PT 60\% x Lysholm & Var const & Var const & $-0,34$ & 0,14 & $-0,26$ & 0,27 \\
\hline PT 180\%/s x Lysholm & Var const & Var const & $-0,28$ & 0,23 & $-0,37$ & 0,11 \\
\hline
\end{tabular}

\section{DISCUSSÃO}

Os resultados do presente estudo demonstraram que os indivíduos do grupo GTP apresentaram maiores déficits no pico de torque extensor comparados com os dos grupos GTF e GC, porém, com significância estatística apenas no teste isocinético a $60^{\circ} / \mathrm{s}(\mathrm{p}<0,001)$.

O pior desempenho do grupo GTP também é demonstrado por maior percentagem dos indivíduos do grupo GTP com déficits $>15 \%$. Esse resultado está de acordo com o de outros autores, que demonstraram maior déficit extensor em pacientes submetidos à reconstrução pela técnica cirúrgica do tendão patelar em curto prazo ${ }^{(26,27)}$. No entanto, ambos os grupos de pacientes submetidos à reconstrução apresentaram déficit extensor significativo em comparação com o grupo controle. Portanto, o déficit no pico de torque extensor não é dependente apenas do tipo de enxerto ou morbidade do local de retirada do enxerto, mas parece ser diretamente influenciado por fatores relacionados com a inibição artrogênica do quadríceps ${ }^{(22,28)}$. Essa inibição seria ocasionada pela destruição dos mecanorreceptores localizados no ligamento cruzado anterior que foi seccionado com a cirurgia ou lesão. Além dessa inibição e consequente atrofia das fibras do quadríceps, há a facilitação da atividade dos isquiotibiais para consequente estabilização dinâmica do joelho ${ }^{(29)}$. Essa teoria do padrão de controle motor facilitando a atividade dos isquiotibiais é observada em estudos que investigaram os déficits no torque flexor no pós-operatório do LCA em pacientes submetidos à reconstrução utilizando ambos os tipos de enxerto, não encontrando diferenças entre grupos ${ }^{(30,31)}$, corroborando os resultados encontrados em nosso estudo, onde não houve diferenças significativas entre os grupos GC, GTP e GTF nos déficits encontrados nos flexores do joelho. No entanto, esses resultados discordam dos de outros autores que observaram déficits significativos no torque flexor em pacientes submetidos à reconstrução do LCA com tendões flexores ${ }^{(26,32)}$. Uma das explicações para os déficits significativos encontrados no torque flexor em pacientes que utilizaram tendões flexores é devida à retirada conjunta dos tendões do semitendinoso e grácil, causando morbidade no local da retirada do enxerto ${ }^{(33)}$. Maior número de trabalhos deve ser realizado para esclarecer se a facilitação dos isquiotibiais ocasionada pela alteração no controle motor favorece a produção de força, mesmo com a retirada do semitendinoso e grácil.

O melhor desempenho do grupo GTF no pico de torque extensor correlacionou-se negativamente com os dois testes de salto monopodal. Essa correlação negativa indicaria que, quanto menores os déficits encontrados no torque extensor, maior era o valor de índice de simetria 
dos membros inferiores (ISM) nesse grupo. Não houve diferença estatisticamente significativa no ISM médio entre os grupos GTF e GTP nos dois testes de salto monopodal, concordando com vários trabalhos que utilizaram testes de salto monopodal para comparar essas duas técnicas cirúrgicas ${ }^{(34,35)}$. Porém, um dado importante e com grande relevância clinica é que menor percentagem de participantes dentro do grupo GTF não obteve o ISM necessário para retorno ao esporte, demonstrando melhor desempenho do grupo como um todo nos testes de salto monopodal comparado com o grupo GTP. Eriksson et $a l^{(36)}$ analisaram o desempenho funcional em 107 indivíduos submetidos à reconstrução do LCA com enxerto autólogo de tendão patelar ( $\mathrm{n}=57)$ e com enxerto autólogo dos tendões flexores $(n=50)$ com tempo médio de 6,5 meses de pós-operatório. Os resultados demonstraram melhor desempenho nos testes de salto monopodal no grupo dos tendões flexores ( $\mathrm{p}<0,01)$. Os autores atribuem essas diferenças ao maior déficit no torque extensor encontrado no grupo dos indivíduos que utilizaram o tendão patelar autólogo como enxerto, fato ocorrido em nosso presente trabalho. Similar ao ocorrido com o pico de torque extensor, ambos os grupos submetidos à reconstrução apresentaram valores do ISM menores do que o grupo GC. Similar ao grupo GTF, houve correlação negativa significativa entre déficit no pico de torque extensor no teste isocinético a $60 \%$ s e salto monopodal a distância no grupo GC. Esses resultados corroboram trabalhos que demonstraram correlação significativa entre pico de torque extensor e desempenho em testes funcionais $^{(7,10,16,18,24)}$. Similar aos achados de Wilk et al, não foi observada, no presente trabalho, correlação significativa entre déficit no pico de torque flexor e testes de salto $^{(18)}$. Apesar da grande importância dos isquiotibiais em fornecer estabilidade articular e trabalhar como antagonista desacelerando o movimento ${ }^{(37)}$, possivelmente o quadríceps desempenhou um papel mais dinâmico na impulsão e aterrissagem dos saltos, correlacionando-se com torque extensor em dois grupos, GC e GTF.

$\mathrm{Na}$ avaliação dos grupos utilizando-se do questionário de Lysholm, o grupo GTP apresentou pontuação significativamente menor que os grupos GTF e GC, conflitando com estudos que empregaram questionários de função para diferenciar o desempenho funcional entre técnicas utilizando o tendão patelar e os tendões flexores e que não encontraram diferenças significativas ${ }^{(38,39)}$. Os menores valores no questionário de Lysholm associados aos déficits no torque extensor isocinético a $60 \%$ s no grupo GTP refletem menor desempenho funcional comparado com os do grupo GTF no presente estudo.
Além do estudo da correlação entre déficits no pico de torque e teste de salto, outro parâmetro avaliado foi a correlação entre frouxidão ligamentar pós- operatória com pico de torque dos extensores e flexores do joelho. Os resultados demonstraram apenas baixa correlação entre déficits no pico de torque e frouxidão ligamentar mensurada através do KT 1000 no grupo GC, GTP e GTF para ambos os grupos musculares analisados (extensores e flexores). A falta de relação entre frouxidão ligamentar e pico de torque encontrada no presente trabalho corrobora trabalhos publicados previamente ${ }^{(10,22,40)}$. Sekiya et al não identificaram correlação significativa entre diferentes níveis de déficits no torque extensor, frouxidão ligamentar e desempenho em testes de salto em pacientes com reconstrução no LCA ${ }^{(10)}$. Ergün et al não encontraram relação entre frouxidão ligamentar e pico de torque isocinético na avaliação de 44 jogadores de futebol sem histórico de lesões do $\mathrm{LCA}^{(40)}$.

Vasconcelos et al analisaram a translação anterior da tíbia, pico de torque isométrico e atividade eletromiográfica em indivíduos com insuficiência do LCA e indivíduos controle ${ }^{(22)}$. Apesar de a translação anterior da tíbia ser significativamente maior no grupo com insuficiência no LCA, não houve diferenças em relação ao pico de torque e atividade eletromiográfica do quadríceps entre os grupos. A falta de correlação entre déficit no pico de torque e frouxidão ligamentar pós- operatória encontrada neste trabalho e em pacientes com insuficiência do LCA no trabalho de Vasconcelos et al pode estar relacionada com a tarefa imposta durante o teste isocinético ou isométrico, não sendo similares aos movimentos que produzam instabilidade, ou seja, movimentos rotacionais ${ }^{(22)}$.

\section{Limitações do estudo}

O principal objetivo deste trabalho foi a correlação entre diferentes instrumentos de avaliação clinica e funcional nos três grupos de sujeitos incluídos no estudo. Como podem ser observados na tabela 1 , diferentes tipos de fixação do enxerto foram utilizados para reconstrução do LCA no grupo GTF. Isso se deve á preferência pessoal dos cirurgiões do departamento de ortopedia no qual os pacientes foram recrutados. Porém, como não observamos diferenças em relação à ADM e frouxidão ligamentar entre os grupos GTP e GTF, isso demonstra que as técnicas cirúrgicas foram adequadas, pois artrofibrose e excessiva frouxidão ligamentar pós-operatória $>10 \mathrm{~mm}^{(41)}$, são fatores diretamente relacionados com a falha na técnica cirúrgica na reconstrução do $\operatorname{LCA}^{(42,43)}$. No entanto, as conclusões a respeito das vantagens do enxerto dos tendões flexores sobre o enxerto do tendão patelar de acordo com a metodologia utilizada neste estudo retrospectivo são limitadas. 


\section{CONCLUSÕES}

De acordo com a metodologia utilizada neste estudo, déficits no pico de torque extensor apresentam correlações significativas com testes de salto monopodal. Este resultado foi observado em dois grupos: GTF e GC.

Na realização de avaliações funcionais em pacientes submetidos à reconstrução do LCA, não é aconselhável a utilização de apenas um instrumento de medida, pois a correlação significativa entre déficit no pico de torque, questionários de função, frouxidão ligamentar e testes de salto monopodal não foi encontrada em todos os grupos, necessitando sempre da combinação entre todos estes parâmetros aqui apresentados.

Se os parâmetros de déficits no pico de torque extensor e índice de simetria entre membros (ISM) dos testes de salto monopodal fossem utilizados como critério de retorno ao esporte sem restrições, ou seja, alta médica, uma percentagem considerável de indivíduos submetidos à reconstrução do LCA com ambas as técnicas cirúrgicas não estariam liberados, mesmo com o tempo mínimo de seis meses de pós-operatório completados, pois demonstraram déficits significativos em ambos os testes.

\section{REFERÊNCIAS}

1. Shimokochi Y, Shultz SJ. Mechanisms of noncontact anterior cruciate ligament injury. J Athl Train. 2008;43(4):396-408

2. Bollen $\mathrm{S}$. Advances in the management of anterior cruciate ligament injury. Curr Orthop. 2000;14(2):325-8.

3. Fu FH, Bennett $\mathrm{CH}$, Lattermann $\mathrm{C}$, Ma $\mathrm{CB}$. Current trends in anterior cruciate ligament reconstruction. Part 1: Biology and biomechanics of reconstruction. Am J Sports Med. 1999;27(6):821-30.

4. De Carlo M, Shelbourne KD, Oneacre K. Rehabilitation program for both knees when the contralateral autogenous patellar tendon graft is used for primary anterior cruciate ligament reconstruction: a case study. J Orthop Sports Phys Ther. 1999;29(3):144-53.

5. Sherman MF, Lieber L, Bonamo JR, Podesta L, Reiter I. The long-term followup of primary anterior cruciate ligament repair. Defining a rationale for augmentation. Am J Sports Med. 1991;19(3):243-55.

6. Daniel DM, Stone ML, Dobson BE, Fithian DC, Rossman DJ, Kaufman KR. Fate of the ACL-injured patient. A prospective outcome study. Am J Sports Med. 1994;22(5):632-44.

7. Sachs RA, Daniel DM, Stone ML, Garfein RF. Patellofemoral problems after anterior cruciate ligament reconstruction. Am J Sports Med. 1989;17(6):760-5

8. Bach BR JR, Jones GT, Sweet FA, Hager CA. Arthroscopy-assisted anterior cruciate ligament reconstruction using patellar tendon substitution. Two- to four-year follow-up results. Am J Sports Med. 1994;22(6):758-67.

9. Pantano KJ, Irrgang JJ, Burdett R, Delitto A, Harner C, Fu FH. A pilot study on the relationship between physical impairment and activity restriction in persons with anterior cruciate ligament reconstruction at long-term follow-up. Knee Surg Sports Traumatol Arthrosc. 2001;9(6):369-78.

10. Sekiya I, Muneta T, Ogiuchi T, Yagishita K, Yamamoto H. Significance of the singlelegged hop test to the anterior cruciateligament-reconstructed knee in relation to muscle strength and anterior laxity. Am J Sports Med. 1998;26(3):384-8.

11. Lephart SM, Kocher MS, Harner CD, Fu FH. Quadriceps strength and functional capacity after anterior cruciate ligament reconstruction. Patellar tendon autograft versus allograft. Am J Sports Med. 1993;21(5):738-43.

12. Sapega AA. Muscle performance evaluation in orthopaedic practice. J Bone Joint Surg Am. 1990;72(10):1562-74

13. Mangine R. Physical therapy of the knee. 2th ed. New York: Churchill Livingstone; 1995 p. $263-89$

14. Fitzgerald GK, Lephart SM, Hwang JH, Wainner RS. Hop tests as predictors of dynamic knee stability. J Orthop Sports Phys Ther. 2001(3);31:588-97.

15. Phillips N, Benjamin M, Everett T, Deursen RMMV. Outcome and progression measures in rehabilitation following anterior cruciate ligament injury. Phys Ther Sport. 2000;1(1):106-18.

16. Greenberger HB, Paterno MV. Relationship of knee extensor strength and hopping test performance in the assessment of lower extremity function. J Orthop Sports Phys Ther. 1995;22(5):202-6.

17. Lephard SM, Perrin DH, Fu FH, Geick JH, McCue FC, Irrgang J. Relationship between selected physical characteristics and the anterior cruciate ligament insuficient athlete. J Orthop Sports Phys Ther. 1992;16(3):174-81.

18. Wilk Ke, Romaniello WT, Soscia SM, Arrigo CA, Andrews JR. The relationship between subjective knee scores, isokinetic testing, and functional testing in the ACLreconstructed knee. J Orthop Sports Phys Ther. 1994;20(2):60-73.

19. Tegner Y, Lysholm J, Lysholm M, Gillquist J. A performance test to monitor rehabilitation and evaluate anterior cruciate ligament injuries. Am J Sports Med. 1986;14(2):156-9.

20. Peccin MS, Ciconelli R, Cohen M. Questionário específico para sintomas do joelho "Lysholm Knee Scoring Scale": tradução e validação para a língua portuguesa. Acta Ortop.Bras. 2006;14(5):268-72

21. Tegner Y, Lysholm J. Rating systems in the evaluation of knee ligament injuries. Clin Orthop Relat Res. 1985;(198):43-9.

22. Vasconcelos RA, Arakaki JC, Simão AP, Oliveira AS, Paccola CJ, Bevilaqua-Grossi D. Análise da translação anterior da tíbia, pico de torque e atividade eletromiográfica do quadríceps e isquiotibiais em indivíduos com lesão do ligamento cruzado anterior em cadeia cinética aberta. Acta Ortop Bras. 2007;15(1):14-8;
23. Kvist J. Rehabilitation following anterior cruciate ligament injury: current recommendations for sports participation. Sports Med. 2004(4);34:269-80.

24. Noyes FR, Barber SD, Mangine RE. Abnormal lower limb symmetry determined by function hop tests after anterior cruciate ligament rupture. Am J Sports Med. 1991;19(5):513-8.

25. Fitzgerald GK, Axe MJ, Snyder-Mackler L. A decision-making scheme for returning patients to high-level activity with nonoperative treatment after anterior cruciate ligament rupture. Knee Surg Sports Traumatol Arthrosc. 2000;8(2):76-82.

26. Feller JA, Webster KE. A randomized comparison of patellar tendon and hamstring tendon anterior cruciate ligament reconstruction. Am J Sports Med. 2003;31(4):564-73.

27. Aune AK, Holm I, Risberg MA, Jensen HK, Steen H. Four-strand hamstring tendon autograft compared with patellar tendon-bone autograft for anterior cruciate ligament reconstruction. A randomized study with two-year follow-up. Am J Sports Med. 2001(6);29:722-8.

28. Bolgla LA; Keskula DR. A review of the relationship among knee effusion, quadriceps inhibition, and knee function. J Sports Rehabil. 2000;9(2):160-8.

29. Fatarelli IFC, Almeida GI, Nascimento BG. Lesão e reconstrução do LCA: uma revisão biomecanica e do controle motor. Rev Bras Fisioter. 2004;8(3):197-206.

30. Adachi N, Ochi M, Uchio Y, Sakai Y, Kuriwaka M, Fujihara A. Harvesting hamstring tendons for $A C L$ reconstruction influences postoperative hamstring muscle performance Arch Orthop Trauma Surg. 2003;123:460-5

31. Tow BP, Chang PC, Mitra AK, Tay BK, Wong MC. Comparing 2-year outcomes of anterior cruciate ligament reconstruction using either patella-tendon or semitendinosus-tendon autografts: a non-randomised prospective study. J Orthop Surg (Hong Kong). 2005;13(2):139-46.

32. Bizzini M, Gorelick M, Munzinger U, Drobny $T$. Joint laxity and isokinetic thigh muscle strength characteristics after anterior cruciate ligament reconstruction: bone patellar tendon bone versus quadrupled hamstring autografts. Clin J Sport Med. 2006;16(1):4-9.

33. Tadokoro K, Matsui N, Yagi M, Kuroda R, Kurosaka M, Yoshiya S. Evaluation of hamstring strength and tendon regrowth after harvesting for anterior cruciate ligament reconstruction. Am J Sports Med. 2004;32(7):1644-50.

34. Corry IS, Webb JM, Clingeleffer AJ, Pinczewski LA. Arthroscopic reconstruction of the anterior cruciate ligament. A comparison of patellar tendon autograft and four-strand hamstring tendon autograft. Am J Sports Med. 1999;27(4):444-54.

35. Ejerhed L, Kartus J, Sernert N, Kohler K, Karlsson J. Patellar tendon or semitendinosus tendon autografts for anterior cruciate ligament reconstruction? A prospective randomized study with a two-year follow-up. Am J Sports Med. 2003;31(1):19-25.

36. Eriksson K, Anderberg P, Hamberg P, Olerud P, Wredmark T. There are differences in early morbidity after $\mathrm{ACL}$ reconstruction when comparing patellar tendon and semitendinosus tendon graft. A prospective randomized study of 107 patients. Scand J Med Sci Sports. 2001;11(3):170-7.

37. Basmajian JV, Deluca CJ. Muscle alive: their functions revealed by electromyography. 5th ed. Baltimore: Wiliams \& Wilkins; 1985. p 93-100

38. Pinczewski LA, Deehan DJ, Salmon LJ, Russell VJ, Clingeleffer A. A five-year comparison of patellar tendon versus four-strand hamstring tendon autograft for arthroscopic reconstruction of the anterior cruciate ligament. Am J Sports Med. 2002;30(4):523-36

39. Shaieb MD, Kan DM, Chang SK, Marumoto JM, Richardson AB. A prospective randomized comparison of patellar tendon versus semitendinosus and gracilis tendon autografts for anterior cruciate ligament reconstruction. Am J Sports Med. 2002;30(2):214-20.

40. Ergün M, Işlegen C, Taşkiran E. A cross-sectional analysis of sagittal knee laxity and isokinetic muscle strength in soccer players. Int J Sports Med. 2004;25(8):594-8

41. Irrgang JJ, Ho H, Harner CD, Fu FH. Use of the International Knee Documentation Committee guidelines to assess outcome following anterior cruciate ligament reconstruction. Knee Surg Sports Traumatol Arthrosc. 1998;6(2):107-14.

42. Kartus J, Magnusson L, Stener S, Brandsson S, Eriksson BI, Karlsson J. Complications following arthroscopic anterior cruciate ligament reconstruction. A 2-5-year follow-up of 604 patients with special emphasis on anterior knee pain. Knee Surg Sports Traumatol Arthrosc. 1999;7(1):2-8

43. Gali JC, Adad MAH, Mod MSB. Causas potenciais de recidiva da instabilidade após reconstrução do ligamento cruzado anterior. Rev Bras Ortop. 2005;40(1/2):52-9. 